\title{
FORMACIÓN DE LOS DOCENTES DE LA URACCAN EN EL ÁREA DE RECURSOS NATURALES
}

Sofía Soza Fuentes ${ }^{[1]}$

Félix Luciano García ${ }^{[2]}$

Neidy Gutiérrez Soza ${ }^{[3]}$

\section{Resumen}

Se abordó la formación de los docentes de la URACCAN en el Área de Recursos Naturales (ARENA), a partir de su perfil profesional y la práctica de sus diferentes estrategias pedagógicas en el aula. Esta área tiene 43 docentes, entre ellos: de tiempo completo, horarios y la coordinación del área. La composición étnica corresponde con $70 \%$ al grupo mestizo, seguido del creol con un $23 \%$; ulwas, garífunas y miskitus con el $2 \%$, respectivamente. Del total de docentes, el $58 \%$ están en proceso de formación pedagógica y el $42 \%$ de ellos no han iniciado estudios de especialización.

La investigación fue cualitativa con enfoque etno-metodológico, tanto en lo que corresponde a la participación de docentes, estudiantes y autoridades del área académica. Se desarrollaron entrevistas, grupos focales y observación directa en las aulas de clase. Relativo a la práctica pedagógicas es importante mencionar que predomina la Pedagogía "depositaria". Entre los factores que han incidido en las prácticas pedagógicas está el abuso del término "libertad de cátedra" y el limitado "acompañamiento participativo", que garantice el cumplimiento del Modelo Pedagógico de URACCAN. Los resultados anteriores, así como el análisis de los reglamentos y normativas internas de la Universidad, conllevan a proponer algunas iniciativas para el diseño de un plan de formación y profesionalización docente.

Palabras Clave: formación, docente, pedagogía, profesionalización.

\section{Summary}

The training of URACCAN teachers in the area of Natural Resources (ARENA) was addressed, based on their professional profile and practice of different teaching strategies in the classroom. This area has 43 teachers, including: full-time, part-time and

\footnotetext{
${ }^{[1]}$ MSc. En Docencia Universitaria y docente de Historia del recinto URACCAN-Bluefields. sofiasfu@yahoo.com

${ }^{[2]}$ MSc. En Docencia Universitaria con mención en Psicopedagogía, tutor de la investigación, Docente Horario de URACCAN-Recinto Bluefields. flgarcia7@yahoo.com.mx

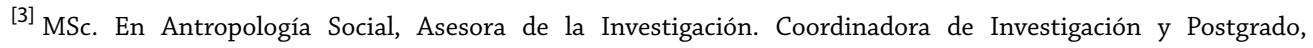
URACCAN-Recinto Bluefields. neidygs@yahoo.com
} 
the coordinator of the area. The ethnic composition corresponds to $70 \%$ from the Mestizo group, followed by Creole with 23\%; Ulwa, Garifuna and Miskitu with 2\%, respectively. From the teachers, $58 \%$ are in the process of pedagogical training and $42 \%$ of them haven't initiated specialized studies.

The research was qualitative with an ethno-methodological approach, corresponding both to the participation of teachers, as well of students and the academic authorities. Interviews, focus groups and direct observation in the classroom were developed. Related to the pedagogical practice is important to mention that the "depository" pedagogy predominates. Among the factors that have influenced teaching practices we can mention the abuse of the term "academic freedom" and limited "participative support", to ensure the compliance of URACCAN Pedagogical Model. The above results and the analysis of the internal rules and regulations of the University are leaded to propose initiatives to design a teacher training and professionalization plan.

Keywords: Training, professor, pedagogy, professionalization.

\section{Introducción}

Los docentes desempeñan un papel fundamental como guías y orientadores en el proceso de enseñanza aprendizaje. Lo anterior conlleva a proponer que la formación integral parte del fortalecimiento de sus capacidades en las diferentes disciplinas: didáctica y pedagogía, filosofía institucional establecida en el Modelo Pedagógico de URACCAN. Fundamentado en ello, el recinto URACCAN-Bluefields desarrolla capacitaciones al inicio de cada semestre académico, los temas se seleccionan a partir de los resultados de las evaluaciones que realizan las autoridades, los estudiantes y desde luego los docentes.

Empero, los esfuerzos anteriores no han producido efectos significativos en los estudiantes, lo cual se refleja en la desmotivación expresada en las evaluaciones estudiantiles sobre el desempeño docente. Por tanto, se presenta un acercamiento sobre la formación pedagógica integral de los docentes en el desempeño de sus funciones, como elemento clave en la búsqueda de calidad y pertinencia.

Para ello, se caracterizan las prácticas pedagógicas, y se valora la percepción de los estudiantes y docentes, alrededor de las prácticas y las capacitaciones, de tal manera, que le permita a la universidad identificar lineamientos para la construcción de una estrategia de formación docente. 


\section{Revisión de literatura}

\section{La formación pedagógica y profesionalización de los docentes}

La formación de los docentes la podemos valorar desde varios puntos de vista, según Cáceres, M. et al (2003), uno de estos, es el aspecto pedagógico metodológico donde se requiere una formación con sólida fundamentación epistemológica de la pedagogía como ciencia. El otro punto de vista, es el de tener características personales como sensibilidad, ética, trascendentalidad e identidad son fundamentales. De igual forma, el maestro requiere el saber pedagógico, cultural, y saber investigar, reflexionar para conocer su contexto histórico, social, político. Finalmente, el docente debe de saber integrar sus conocimientos y habilidades para proyectarse a la comunidad.

La Conferencia mundial de Educación Superior de la Unesco (2009), plantea que: "las instituciones de educación superior deben de invertir en la formación de sus docentes, para cumplir nuevas funciones de enseñanza y aprendizaje que evolucionan constantemente. También, la importancia de atraer y retener al personal docente y de investigación calificado, talentoso y comprometido".

Cáceres, M, et al (2003), plantea que la profesionalización del docente tiene diferentes estadios, entre los que se destacan al profesor como trabajador, artesano, artista y, profesional. Esta afirmación establece que el profesor no es propenso a la mecanización y que debe estar comprometido con la autoreflexión y el análisis de las necesidades de los estudiantes. El docente profesional, debe analizar desde tres criterios fundamentales: 1) La existencia de un cuerpo de conocimientos provenientes de la investigación y la elaboración teórica, 2) Compromiso ético de la profesión y 3) Registrarse por una normativa interna de autocontrol por parte del colectivo profesional.

Por lo anterior, la formación de los docentes requiere de profesionalización. Es decir, hacer de la docencia una actividad profesional. Esto implica, prepararlos pedagógicamente 1) Dirigir el proceso de enseñanza-aprendizaje de la disciplina que desarrolla, 2) Investigar el proceso para su perfeccionamiento, 3) Incrementar su autonomía y control del trabajo propio, 4) Poseer un cuerpo de contenidos científicos consistentes y a la vez una ética y compromiso.

\section{El desempeño del docente universitario}

Los factores que inciden en el desempeño son la formación inicial, el desarrollo profesional, los niveles salariales, de salud, auto-estima, compromiso profesional y clima institucional, incentivos y la evaluación del desempeño (Robalino, M 2006: 65). Sin embargo, los ambientes pedagógicos, también se consideran factores que pueden incidir en dicho desempeño, y según Duarte, J, (2003) son parte de la naturaleza de las actividades académicas que desarrollan los docentes. 


\section{La pedagogía y modelos de formación}

La pedagogía en la universidad debe encontrar su fundamento en el carácter formativo que tiene cada institución de la Educación Superior, y no debe consistir en repeticiones de textos para ser examinado. Expresa Araujo, Freire (2004: 24), que los educadores deben ser competentes, tanto en los ámbitos científico, técnicos y filosóficos.

Por otro lado, los modelos de formación del profesorado según Cáceres et al (2003), son recursos para el desarrollo y fundamentación científica de la enseñanza, la formación docente se concibe como el proceso permanente de adquisición, estructuración y reestructuración de conocimientos, habilidades y valores para el desempeño de la función docente. La formación es continua y se lleva a cabo a lo largo de toda la práctica docente, como eje formativo estructural.

Los modelos de formación del profesorado se basan en dos tipos, el modelo teórico, cuyo propósito es formar profesionales capaces de responder a exigencias que se les plantee en cualquier situación académica y el modelo critico-reflexivo que forma parte de un movimiento de reeducación curricular y de enseñanza más amplia, que asume la idea, como eje central en la formación del profesor investigador.

\section{Materiales y métodos}

La investigación, correspondió al tipo cualitativa con un enfoque etnometodológico, en la comprensión y explicitación de la practica pedagógica de los docentes desde los procesos institucionales e instituyentes creados a partir del quehacer cotidiano (Merlino, A. 2009:57).

La población de estudio y los grupos seleccionados correspondió a los docentes de tiempo completo y horarios (para un total de 5), estudiantes ( 15 en grupos focales y en las aulas de clases) y autoridades universitarias de las dos carreras del área de Recursos Naturales (Ing. Agroforestal y Licenciatura en Ecoturismo). Así mismo, se tomaron como informantes claves la responsable de Biblioteca y graduados del área de Recursos Naturales.

Se realizaron entrevistas, grupos focales, observaciones directas y revisión documental de informes relacionados al quehacer docente del área. El análisis y procesamiento de la información se realizó en base a la matriz de descriptores diseñados y en cumplimiento a la ética.

\section{Resultados y discusión}

Caracterización de los elementos de formación pedagógica de los docentes en el área de Recursos Naturales. 
En el Área de Recursos Naturales (ARENA), en el período de estudio (I Semestre del año académico 2010) se constató que el $9 \%$ de los docentes están contratados a tiempo completo, (incluye al coordinador de carrera y algunos funcionarios del recinto); el $91 \%$, son de tiempo horario; sin embargo, estos últimos solamente tienen la función de facilitar docencia, según el programa de asignatura y contrato laboral.

Lo anterior, establece la brecha existente entre el tipo de contratación y la no existencia de otras formas establecidas en el reglamento docente. Esta modalidad de contratación (horario de cátedra y tiempo completo) y con el predominio de docentes de cátedra, limita la atención integral y la construcción de un Modelo de Educación Superior Comunitaria, ya que los docentes universitarios deben desempeñar funciones de investigación, docencia y extensión. De igual manera, la permanencia en la contratación de docentes de cátedra (horarios) está ligada a factores externos de la institución, situación que dificulta la inversión en la profesionalización docente. Esta dificultad es reconocida por la autoridad del recinto cuando expresa:

En gran medida nuestro personal docente es horario y eso nos crea ciertas dificultades, dado a que podríamos decir que hay constantes cambios de docentes. En la medida de lo posible logramos mantener una planta bastante estable de docentes (Zarifeth Bolaños Chow, Vicerrectora del Recinto, 2010).

Así mismo, según Cáceres M. (2003), el profesor universitario debe de tener compromisos éticos, lo cual también se puede traducir a una identidad institucional reforzada. Por lo que el docente de cátedra, por su propia forma de contratación y permanencia en la institución, puede ser actor complejo de fortalecer.

Por otro lado, el reglamento docente destaca la presencia de los docentes con tiempo completo y medio tiempo, entre los que señala el docente auxiliar, asistente, asociado y titular. Los anteriores, más allá de la contratación, responden a las funciones y retos del docente universitario actual (docencia, investigación y extensión). Estas categorías, a pesar de estar normadas, todavía no se desarrollan; no obstante, la autoridad del recinto, consideran que es un elemento clave para la construcción de un modelo de profesionalización docente.

En relación a la composición étnica, predomina el grupo mestizo con un 70 \% de docentes, seguido del grupo creole con un $23 \%$ y ulwas, garífunas y miskitus con el 2 $\%$, respectivamente. Esta situación, no limita la calidad de la docencia; empero, las aulas de clase son multiculturales y necesitan atención integral desde una cosmovisión indígena y afrodescendiente. Esto también es tomado en cuenta por los docentes mestizos, ya que en la práctica son múltiples las barreras que enfrentan. 
De igual forma, los docentes indígenas consideran que es necesario y urgente que la universidad disponga de una planta docente indígena. Esto lo reafirma el docente indígena de URACCAN, Melvin James, cuando expresa que:

La lengua es la llave de las prácticas interculturales en el aula de clases. Es la forma de expresar los sentimientos, las ideas [...] es el medio de expresar y conocer la cosmovisión de los pueblos [...] es una de las formas de cómo relacionamos los conocimientos endógenos con los exógenos... fortalecería los conocimientos científicos técnicos de los estudiantes, ya que se facilita una mejor estrategia de comunicación en la interacción docente-estudiante.

En relación a la distribución de género: el $54 \%$ de los docentes son mujeres, y el $46 \%$ son hombres. Esta distribución se considera balanceada. De igual manera, en las diferentes acciones de capacitación y formación docente participan docentes de tiempo completo y horario, tanto de mujeres y hombres.

En referencia a la calidad en la profesionalización se han desarrollado diferentes estrategias, entre estas las capacitaciones realizadas al inicio de cada semestre, maestrías y postgrados en docencia universitaria. Estos espacios de formación se facilitan a los docentes de tiempo completo y horario, que tengan o no formación en docencia.

También, se puede precisar que el $58 \%$ están en proceso de profesionalización, puesto a que han iniciado su formación docente, principalmente cursando la Maestría en Docencia Universitaria (5) y el postgrado en Docencia Universitaria con mención en las TIC's (16), y otros ya graduados de la maestría en Docencia Universitaria con mención en Psicopedagogía (4). En cambio el 42 \% (18 docentes), no han tenido ni iniciado ningún tipo de formación pedagógica.

En cuanto a los docentes que están finalizando la Maestría en Docencia Universitaria, dos de ellos, tienen una formación inicial en recursos naturales, son Ingenieros Agrónomo e Ingeniero en Pesca. Mientras que los otros tres son docentes con formación en Ciencias de la Educación (Matemática e Historia). En el postgrado en Docencia Universitaria con mención en TIC's, participan mayoritariamente docentes sin formación pedagógica. Es decir, docentes cuya formación inicial es científica-técnica en recursos naturales (Ingenieros agrónomos, zootecnistas, ecólogos, ecoturismo, etc.).

Por otro lado, el restante $42 \%$, en su mayoría son profesores de las asignaturas técnicas, por lo cual es necesario continuar con el proceso de formación pedagógica para fortalecerlos como docentes universitarios profesionales.

Así mismo, tomando en consideración los planteamientos de Cáceres et al (2003), si los programas de formación pedagógica no incluyen elementos que le den salida a 
la formación pedagógica, ni científica-técnica con valores y principios, solamente se forman docentes, pero no se profesionalizan a los docentes. Es decir, no es suficiente conocer y manejar herramientas pedagógicas y didácticas, hace falta profundizar y promover la formación integral de los profesores, para ampliar sus capacidades disciplinarias y didácticas, así como estimular el cambio de la actuidad positiva que responda al modelo de universidad comunitaria e intercultural.

Por ello, se hace necesario asegurar que el profesor sea especialistas en su materia, con dominio tanto del conocimiento, métodos, procedimientos y enfoques de la disciplina que enseña; que se actualice constantemente y que se acerque al conocimiento de su especialidad, de tal manera que pueda motivar de manera constante el interés de los estudiantes.

\section{Ambiente pedagógico de los docentes de ARENA}

Es en el aula de clase donde se comparten conocimientos, sentimientos y experiencias. En la URACCAN, estas relaciones tienen una característica singular, ya que los salones de clases son centros de convergencia multiétnica y multicultural. Esta multiculturalidad, demanda que los conocimientos se construyan en base al respeto mutuo, autoestima, tolerancia, búsqueda y consolidación de la identidad étnica, entre otros aspectos fundamentales de interculturalidad, para el desarrollo profesional de los estudiantes.

En lo que respecta a la biblioteca, esta es considerada una de las instancias que debe facilitar condiciones necesarias para ampliar, desarrollar y consolidar los conocimientos de docentes y estudiantes, ya que pone a la disposición de la comunidad educativa todo el material bibliográfico. Sin embargo, la falta de bibliografía y poco acceso a la tecnología de la información es de suma preocupación, ya que no permite construir una cultura de auto aprendizaje y de excelencia.

Los docentes expresan que para la carrera de Ecoturismo, no hay libros que respondan al perfil, por lo que tienen que buscar información y adecuar o adaptarla al contexto social, ambiental y cultural de la región. Por otro lado, los maestros de tiempo completo expresan que:

Las políticas internas de la biblioteca afectan el quehacer de los estudiantes y los docentes, no hay espacios para lectura, no hay espacios de discusión, el no poder sacar copias de algunos libros. Las políticas no están incentivando ni a los docentes, ni a los estudiantes a visitarla.

Por consiguiente, la responsable de la biblioteca plantea que a pesar de tener un edificio nuevo y grande, no está estructurada con los requerimientos necesarios que debe tener un espacio tan importante para la academia. De igual forma, la compra de 
bibliografía actualizada está condicionada al limitado presupuesto. Estos datos son reafirmados por la responsable de la biblioteca del recinto cuando manifiesta que: "Los docentes no tienen cultura y hábito de revisar la bibliografía recomendada en los programas de las diferentes carreras y muy pocos la visitan. Esto es igual en el caso de los estudiantes".

La funcionaria de la biblioteca expresa que persisten debilidades que hay que superar, como la falta de coordinación y comunicación con las áreas académicas y las instancias superiores para la búsqueda de respuestas a las dificultades y demandas de la academia. Se observa un incremento en la adquisición de libros y revistas, pero estos no corresponden a los sugeridos en los programas de estudio. De la misma manera, los estudiantes no cuentan con laboratorios instalados que les permitan comprobar los conocimientos teóricos. Hay que destacar que en este año, se hizo una adquisición de equipos de laboratorios que permitirá a las próximas generaciones tener mejores estándares de calidad en los procesos educativos.

En lo que respecta a horas prácticas, los programas de Ingeniería Agroforestal tienen como requisitos de graduación el cumplimiento de las prácticas pre-profesionales, las cuales corresponden a 240 horas agrupadas en 16 créditos; donde cada crédito es el equivalente a 15 horas. A pesar de lo anterior, el recinto no cuenta con una finca didáctica para las prácticas de campo de sus estudiantes. Ante ello, los maestros de esta carrera hacen esfuerzos para cumplir con las horas prácticas, coordinando el trabajo en diferentes fincas del municipio. Al respecto los docentes expresan que: "Los estudiantes apenas cumplen un $25 \%$ de las clases prácticas de los programas de estudios..."

(Docente horario, 2010).

En la carrera de Licenciatura en Ecoturismo, el comportamiento es similar, el diseño curricular contempla que el recinto entre otras cosas cuenta con hotel, restaurante y comedor entre otros aspectos, pero ellos desconocen las razones por la cual estos no son accesibles para las prácticas de los estudiantes.

Respecto a las prácticas pedagógicas planteadas en el Modelo Pedagógico de URACCAN se establece que el docente debe ser respetuoso de la diversidad étnica y de género, vinculando la ciencia con los saberes ancestrales. No obstante, en las observaciones hechas en las aulas de clase, se comprueba que la mayoría de los docentes desarrollan sus clases de manera tradicional.

Resultado de las observaciones se identificaron dos tipos de maestros: el autoritario, caracterizado por ser exageradamente receloso de la disciplina rígida, aplica reglamento sin un mínimo de flexibilidad, desconfiando de las capacidades de sus estudiantes e impidiendo la participación activa en el aula de clases. 
Por otro lado, el maestro consentidor, que carece de una planificación de sus clases, hace énfasis en la actividad independiente de los estudiantes, como mecanismo para justificar la displicencia de su actitud. Utiliza solamente la pizarra y de vez en cuando algunos medios de enseñanza. Sus clases son monótonas y hace hincapié en el conocimiento memorístico. No construye procesos de razonamiento y análisis. Los estudiantes lo perciben solamente como receptor de conocimientos y no se sienten en libertad para expresar sus opiniones libremente. Este tipo de maestro es el que predomina en el área.

Estas prácticas son visibilizadas por el estudiantado cuando expresan que:

Los maestros cuando nos dan las clases, sólo nos dictan de los libros o de sus computadoras los temas que nos dan [...] explican los contenidos en la medida que hacen los dictados [...] y explican los temas cuando les hacemos preguntas (Estudiantes de Lic. Ecoturismo, 2010).

Así mismo, los docentes están aplicando una Pedagogía “depositaria”, llamada así por Paulo Freire, por la aplicación de técnicas unidireccionales, donde los estudiantes son depositarios de conocimientos por los maestros, aplicando una Pedagogía de respuesta. Según Freire la Pedagogía de respuestas, son aquellas estrategias didácticas donde el maestro es el que tiene el control del aula y de los estudiantes, es el único que hace las preguntas.

Estas concepciones educativas de Paulo Freire, que no contradicen las del Modelo Pedagógico de URACCAN, nos llevan a concluir que la práctica pedagógica de la mayoría de los maestros de ARENA, no son adecuadas según lo que establece la universidad.

Cuadro No. 1. Resumen de las prácticas pedagógicas de los docentes de ARENA

\begin{tabular}{|c|c|}
\hline $\begin{array}{c}\text { Práctica pedagógica según el Modelo } \\
\text { Pedagógico de la URACCAN }\end{array}$ & $\begin{array}{l}\text { Prácticas pedagógicas que desarrollan en } \\
\text { las aulas de clase los docentes en ARENA }\end{array}$ \\
\hline $\begin{array}{l}\text { - Facilitan, promuevan y generen la reflexión, la } \\
\text { discusión y la búsqueda de alternativas crea- } \\
\text { tivas. } \\
\text { - Ejercitan de manera combinada la docencia y } \\
\text { la investigación. } \\
\text { - Orientan hacia la formación humanista comu- } \\
\text { nitaria. } \\
\text { - Orientan a la formación de conciencia ética } \\
\text { que establece un nexo con la sociedad. } \\
\text { - Sistematizan y difunden ideas, conocimientos, } \\
\text { experiencias que se generen en la vida interior } \\
\text { de la universidad. Con capacidad investigativa } \\
\text { y de transferencia tecnológica. }\end{array}$ & $\begin{array}{l}\text { - Poca promoción de espacios de discusión y } \\
\text { reflexión, poco creativos, sus prácticas pedagó- } \\
\text { gicas la consideran rutinarias según lo expresa- } \\
\text { do por los estudiantes. } \\
\text { - Poco espíritu investigativo, según opinión de } \\
\text { la coordinadora del área de Investigación y } \\
\text { Postgrado y Educación Continúa. } \\
\text { - Hay poca discusión y análisis de los contextos } \\
\text { comunitarios, especialmente aquellos con la } \\
\text { vida académica e institucional en sus princi- } \\
\text { pios, misión y visión. }\end{array}$ \\
\hline
\end{tabular}


Esta situación es producto del desconocimiento de la importancia de su función y falta de identificación con la filosofía institucional. Este escenario más la falta de acompañamiento activo, constante y participativo de las instancias que garanticen el cumplimiento del Modelo Pedagógico de URACCAN, lleva a que algunos maestros tengan concepciones erradas de lo que se conoce como "Libertad de Cátedra".

\section{Percepción sobre la práctica pedagógica y la profesionalización docente}

Cáceres (2003), plantea que para mejorar la calidad de la enseñanza aprendizaje es necesario la trasformación del pensamiento, los sentimientos de los profesores, acompañados de la calidad del personal docente, de los programas de estudio, de los estudiantes, de la infraestructura y del ambiente universitario.

Partiendo de estas consideraciones, se hace una descripción de la percepción que tiene la comunidad educativa sobre las prácticas pedagógicas y la profesionalización de los docentes. Esta percepción inicia desde la perspectiva de las autoridades del recinto, tomando como referencia a Zarifeth Bolaños Chow, quien ha expresado: "el diseño de la Maestría en Docencia Universitaria y los Postgrados en Docencia, son los productos más tangibles de los resultados de los lineamientos y políticas institucionales de la universidad..." [...] Además, plantea que a inicios de cada semestre académico se desarrollan capacitaciones para los docentes. Estas se construyen a partir de: “...la autoevaluación de los maestros, la evaluación que hace el coordinador de la carrera después de cada corte parcial, así como de las evaluaciones que hacen los estudiantes a sus maestros en cada semestre académico" (Vicerrectora del Recinto URACCAN, Bluefields; 2010).

Hay que mencionar además, que las autoridades tiene la plena confianzas que estas acciones de formación pedagógica en los maestros deben traducirse en mejores resultados en cuanto a permanencia de los estudiantes en las aulas de clases, calidad de los procesos de enseñanza aprendizajes, mayor apropiación y manejo del modelo pedagógico, entre otros logros académicos.

Así mismo, el coordinador del Área de Recursos Naturales, ARENA, considera que:

... a pesar de que la mayoría de los docentes de las asignaturas científicas, su formación profesional proviene de contextos culturales diferentes, mayoritariamente monolingüe, hacen esfuerzos para dar lo mejor de ellos a pesar de los pocos recursos económicos, y limitada bibliografía para complementar los conocimientos de sus estudiantes. (MSc. Juan Mendoza, Coordinador de ARENA, 2010).

Los docentes tanto horarios y de tiempo completo, tienen sus propias percepciones de la formación profesional y pedagógica en el recinto. Consideran que las 
capacitaciones que se imparten a inicio de cada semestre, son consideradas como charlas, poco interesantes y muy repetitivas; causando desmotivación, sobre todo a docentes que tienen años de experiencia.

Pero también tienes expectativa en relación a los contenidos desarrollados en las capacitaciones. “...es importante seguir profundizando el modelo pedagógico para los que lo conocen, pero también hay docentes que muy poco saben de él”. (Docente horario, 2010).

Porque trata de comprenderlo, (Modelo Pedagógico) lo leen, pero a la hora de dar las clases, se les escapa como debe ser el tratamiento que se le debe dar a los estudiantes que no son mestizos, a la hora de la evaluación, también, pero no quieren aprobar un alumno sólo por ser de otra comunidad con otras características étnicas. (Docente tiempo completo, 2010).

Estas aseveraciones reflejan la preocupación de muchos docentes por la identificación con la filosofía institucional, desde la perspectiva de las aulas multiculturales. De igual forma, los estudiantes consideran que sus conocimientos son teóricos, porque no tienen espacios idóneos para sus prácticas y giras de campo.

Los docentes les han suministrado conocimientos básicos para el desempeño en el campo laboral. Las asignaturas les han generado conocimientos básicos. Hace falta la práctica, esto es una limitante, porque solo la teoría no es suficiente para ir al campo laboral (Estudiante de Ecoturismo, 2010).

\section{Elementos para la construcción de un plan de profesionalización}

El plan de formación y profesionalización docente universitario de la URACCAN va más allá del postgrado y la maestría en docencia universitaria, pedagogía o didáctica, al considerar un proceso de formación continua y de constante evaluación del desempeño docente. A continuación, se detallan algunos elementos:

En relación al tiempo (duración): el plan de formación debe ser permanente y considerar las categorías establecidas en el reglamento docente, desde el docente auxiliar hasta el titular.

Estrategias metodológicas: la formación debe ser teórica-practica, incluyendo y respetando los saberes tradicionales planteados en el modelo pedagógico.

Seguimiento y monitoreo: es constante por parte de las instancias correspondientes, entre estas Secretaría Académica. Sin embargo, se considera necesario crear un área metodológica y/o un asesor metodológico que acompañe este proceso. De igual 
manera, la universidad debe iniciar un proceso de evaluación del desempeño docente que se logre articular con el plan de formación y profesionalización.

Abordaje de temáticas: docencia universitaria, pedagogía, didáctica, currículo, evaluación educativa y acreditación, metodología de la investigación, filosofía institucional, ética y la especialidad científica técnica de acuerdo a la carrera que imparte.

\section{Conclusiones}

Para la universidad el profesor es un actor clave, quien representa y hace realidad la propuesta educativa cuando materializa los fines y objetivos de la misión y visión del modelo de universidad comunitaria e intercultural.

Los temas de capacitación a los docentes, han sido el resultado de las evaluaciones que cada corte de semestre se hace con estudiantes, docentes y autoridades académicas del recinto sobre el desempeño del personal docente. Sin embargo, hace falta profundizar más en la especialización de los profesores en la materia que imparten, para que los mismos dominen tanto el conocimiento como los métodos, los procedimientos y los enfoques de la disciplina que imparten.

En el recinto se ha desarrollado dos postgrados, una maestría y un postgrado en docencia universitaria con el propósito de preparar a los docentes en el manejo conceptual de sus funciones. Es importante diseñar e implementar un plan de formación orientado a las modalidades de contratación y categorización de los docentes.

La planta docente es mayoritariamente horaria, por lo que para la profesionalización se debe ampliar la contratación de más docentes con tiempo completo, de tal forma que puedan ejercer las funciones de docencia, investigación y extensión.

En relación a las prácticas pedagógicas, en su mayoría se caracterizan por desarrollar procesos de enseñanza aprendizaje, que se conciben como educación depositaria según Paulo Freire, por lo que no responden a lo establecido en el Modelo Pedagógico de la Universidad.

El personal docente es relativamente joven, se deben de potenciar y fortalecer para futuras contrataciones. De igual forma, considerar la contratación de docentes indígenas y afrodescendientes.

Es necesario mejorar las condiciones que generan un ambiente pedagógico favorable para el proceso de enseñanza aprendizaje, tales como la biblioteca, el acceso a internet y paquete informático, los laboratorios y fincas para el desarrollo del trabajo pre-profesional. 
Son necesarios los acompañamientos constantes y participativos para mejorar el proceso de enseñanza aprendizaje, principalmente en las asignaturas de la especialidad. Razón por la cual, se requiere de asesoría metodológica que trabaje con la Secretaría Académica y coordinaciones, para facilitar el seguimiento, control y asesoría metodológica a los docentes.

\section{Lista de referencias}

Araujo Freire, A. M. 2004 (Coord.). La Pedagogía de la liberación de Paulo Freire. Editorial GRAO, de IRIF, SL.

Merlino, A. 2009 (Coord.). Investigación cualitativa en ciencias Sociales. Cengage learning. Argentina.

Cáceres Meza, Maritza (2003). La formación pedagógica de los profesores universitarios: una propuesta en el proceso de profesionalización docente. Universidad de Cienfuegos, CUBA. Revista Iberoamericana de Educación. Recuperado el 15 de Marzo del 2010 de http://www.rieoei.org/deloslectores/475Caceres.pdf

Duarte, J. (2003). Ambiente de Aprendizaje, una aproximación conceptual, Universidad de Antioquia, Colombia. Revista Iberoamericana de Educación. Recuperado el 12 de marzo del 2011 en http://www.rieoei.org/deloslectores/524Duarte.PDF

Robalino, M. (2006). Carrera y evaluación docente en americe Latina: una mirada desde la perspectiva renovadora de la profesión docente. UNESCO. Recuperadoel12deJuniodel201ode: http://books.google.com.ni/books?id=Vq VJ19eCdZMC\&pg=PA143\&dq=Robalino, + M. $+2006 . \&$ hl=es\&cd=5\#

UNESCO (2009). Conferencia Mundial sobre la Educación Superior: Las nuevas dinámicas de la educación superior y de la investigación para el cambio social y el desarrollo.

UNESCO (1996). Conferencia Mundial sobre Políticas y Estrategias para la Trasformación de la Educación Superior en América Latina y el Caribe.

URACCAN (2004). Modelo pedagógico de URACCAN. Managua- Nicaragua.

URACCAN (2004). Diseño Curricular de la carrera Ingeniería Agroforestal (URACCAN), Bluefields, RAAS, Nicaragua.

URACCAN (2006). Diseño Curricular de la carrera de Licenciatura en Ecoturismo (URACCAN). Bluefields, RAAS, Nicaragua. 
URACCAN (2010). Informe de Gestión Institucional Recinto Bluefields, RAAS-Nicaragua.

URACCAN (2003). Revista Universitaria Caribe $N^{o}$ 9, RAAS, Nicaragua.

URACCAN (2004). Modelo de Universidad Comunitaria e Intercultural. Nicaragua.

URACCAN (2007). Reglamento Del Docente. Managua, Nicaragua. 